\title{
Nested structures approach for bulk 3D negative index materials [invited]
}

\section{Andryieuski, Andrei; Malureanu, Radu; Lavrinenko, Andrei}

\section{Published in:}

Proceedings, ICTON

Link to article, DOI:

10.1109/ICTON.2009.5185296

Publication date:

2009

Document Version

Publisher's PDF, also known as Version of record

Link back to DTU Orbit

\section{Citation (APA):}

Andryieuski, A., Malureanu, R., \& Lavrinenko, A. (2009). Nested structures approach for bulk 3D negative index materials: [invited]. In Proceedings, ICTON (pp. Th.A1.2). IEEE. https://doi.org/10.1109/ICTON.2009.5185296

\section{General rights}

Copyright and moral rights for the publications made accessible in the public portal are retained by the authors and/or other copyright owners and it is a condition of accessing publications that users recognise and abide by the legal requirements associated with these rights.

- Users may download and print one copy of any publication from the public portal for the purpose of private study or research.

- You may not further distribute the material or use it for any profit-making activity or commercial gain

- You may freely distribute the URL identifying the publication in the public portal 


\title{
Nested Structures Approach for Bulk 3D Negative Index Materials
}

\author{
Andrei Andryieuski, Radu Malureanu, Andrei V. Lavrinenko \\ Technical University of Denmark, Ørsteds Plads, Building 343, Kgs. Lyngby,DK-2800, Denmark \\ Tel: (45) 4525 6392,Fax: (45) 4593 6581,e-mail:alav@fotonik.dtu.dk
}

\begin{abstract}
We investigate two generic conceptual ideas to obtain bulk 3D negative index metamaterials, which exhibit isotropic properties. One design is a traditional design of 3D wires ended with metallic plates. Another design is based on the nested structures approach, when one element providing magnetic response is inserted into another design with negative dielectric constant. Both constitutive elements possess cubic symmetry, thus we preserve it for the whole unit cell. The concepts can be applied for any frequencies; however, we are targeting optical and $\mathrm{THz}$ ranges. We report on numerical characterization of these particular designs finding negative index behaviour in "split-cube-in-cage" and "split-cube-in-carcass" designs. The latter design can be considered as reasonable candidate for isotropic bulk metamaterial showing good results in isotropy of effective properties and their fast convergence with the thickness of a sample.
\end{abstract}

\section{INTRODUCTION}

Bulk isotropic negative index materials (NIMs) [1] are required to implement superresolution lens [2] and nanocouplers [3]. Few 3D isotropic designs have been proposed but they work mostly in the microwave regime. There is a lack of isotropic bulk NIMs for optics. In this work we propose a bulk isotopic negative index material design and the nested structures approach to construct isotropic NIM.

To be more specific the standard approach to construct NIM is to combine negative permeability $\mu$ part with negative permittivity $\varepsilon$ part [4]. Usually such combination as well as usage of planar technology for fabrication destroys isotropy of the system. For example, the best so far design in optics - the fishnet structure [5] has good optical properties (negative refractive index and high transmission) but is anisotropic. Our nested structures approach allows obtaining negative index while preserving cubic symmetry and thus saving isotropy of the system.

In this work we summarise our results on NIM design investigation and follow the evolution of structures, which has led to the Split Cube in Carcass (SCiC) design. SCiC shows refractive index -1.5 and maximal figureof-merit 2.2 at telecom frequency $187 \mathrm{THz}$. Fine convergence of the effective parameters with the number of layers and band structure allow to regard SCiC as bulk isotropic NIM.

\section{METHODOLOGY}

To retrieve the effective parameters of NIM we used the standard method [6] based on the calculation of Sparameters (amplitude and phase reflection and transmission coefficients). For simulations of the S-parameters we used the CST Microwave Studio software in the frequency domain regime. For the reflection-transmission of a TE-polarized (electric field along y-axis) plane wave propagating along the $z$-axis the perfect magnetic conductor (zero tangential component of magnetic field), the perfect electric conductor (zero tangential component of electric field) and open boundary conditions were chosen for $\mathrm{x}-, \mathrm{y}-\mathrm{and} \mathrm{z}$ - axes respectively.

We proved the isotropic response of our system by calculating bands dispersion propagating in three principle directions of the cubic unit cell. Unfortunately it is out of the CST Microwave Studio functionality to simulate the $\mathrm{SCiC}$ band structure taking into account material dispersion, e.g. as a Drude metal, so we performed the simulation for fixed permittivity -140 . Losses were also omitted $(\operatorname{Im} \varepsilon=0)$.

\section{METAMATERIALS DESIGNS}

\subsection{Metamaterials' designs}

We investigated three metamaterial (MTM) designs (Fig. 1): Cross and Plates (CaP), Edge Split Cube in Cage (ESCiC) and Split Cube in Carcass (SCiC). The MTMs consist of metal parts embedded in silica (refractive index $n=1.5$ ). Metal is regarded within the Drude model with plasma frequency $\omega_{\mathrm{p}}=1.39 \cdot 10^{16} \mathrm{rad} / \mathrm{s}$ and collision frequency $v_{\mathrm{c}}=1.68 \cdot 10^{13} \mathrm{~s}^{-1}$ resembling the properties of bulk silver. The cell constant was in all cases equal to $a=250 \mathrm{~nm}$ that corresponds to the highest frequency $f=c /(4 a n)=200 \mathrm{THz}$, where the MTM can be approximated by a homogenous material model.

The Cross and Plates structure (Fig. 1A) consists of six square plates of size $175 \mathrm{~nm}$ and thickness $20 \mathrm{~nm}$. The centre of the plate is at $100 \mathrm{~nm}$ from the unit cell centre. All the plates are connected with square $20 \mathrm{~nm}$ thick rods. This model is similar to regarded in [7], where metallic plates were applied to diminish the spatial dispersion of the wire medium. 
The Edge Split Cube in Cage (Fig. 1B) is a first realization of the nested structures concept. It consists of two parts. The inner part (Edge Split Cube) is represented by six non-touching square plates of size $126 \mathrm{~nm}$ and thickness $20 \mathrm{~nm}$ forming a cube with removed edges. The centre of the plate is at $64 \mathrm{~nm}$ from the unit cell centre. The outer part (Cage) defines the unit cell border and consists of 6 connected perforated plates made from square cross-section wires having a thickness of $20 \mathrm{~nm}$ equidistantly placed along the edge of the unit cell.

The Split Cube in Carcass (Fig. 1C) is the extension of the nested ESCiC design with an attempt of simplification. The inner part (Split Cube) is the hollow cube of size $160 \mathrm{~nm}$ and thickness $20 \mathrm{~nm}$ with the $5 \mathrm{~nm}$ slits in the middle of the facets. The outer part (Carcass) defines the unit cell border. It is made from six $20 \mathrm{~nm}$ thick plates with $110 \mathrm{~nm}$ square hole in each facet.
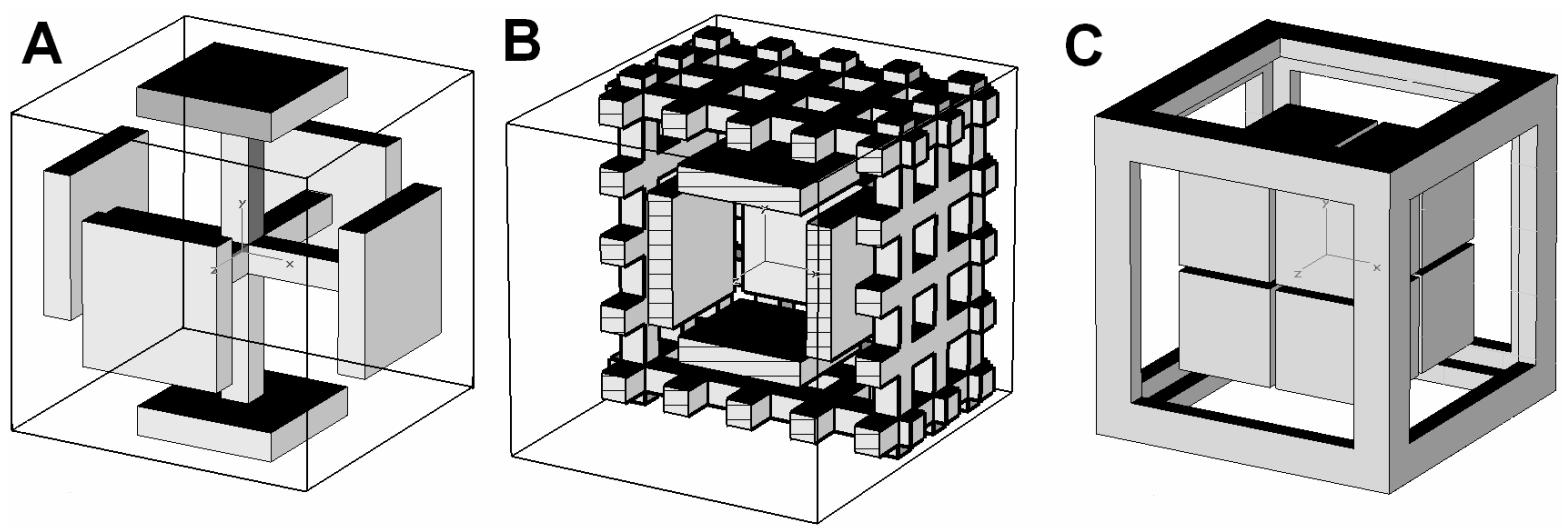

Figure 1. Considered metamaterial designs: Cross and Plates (A), Edge Split Cube in Cage (B) and Split Cube in Carcass (C). All parameters are given in the text.

\subsection{Metamaterials' effective parameters}

First we extracted the effective parameters for the CaP design. It does not show negative refractive index (Fig. 2A). The reason for that became clear when we retrieved effective dielectric and magnetic constants. Permittivity (Fig. 3A) is positive in the whole range of interest, and a non-physical resonance with negative imaginary part at $160 \mathrm{THz}$ is observed. That corresponds to the electric dipole oscillations. The Plates makes a sort of split-ring resonator, which leads to resonant behaviour. Field simulations prove that magnetic resonance occurs here, and there is a range of negative permeability (Fig. 4A). However due to strong resonant response of the system the homogeneity limit is certainly broken.

It became obvious that we do not have negative permittivity because the metallic wires (Cross) are not long. We connected the crosses of the neighbour unit cells passing the wire through the Plates. That led to negative permittivity, but, in turn, negative magnetic properties were lost in this case.

To solve this problem we formulated nested structures approach: to take electric negative and magnetic negative subsystems with cubic symmetry and put one into another. In this case we save isotropy because the entire system has cubic symmetry as well. We also switched to the extended $\operatorname{Re} \varepsilon<0$ range covered by the Drude model instead of narrow electric resonances. The electric negative subsystem should be very long so we should place it on the periphery of the unit cell and put magnetic meta-atom inside.

The first NIM obtained with nested structures approach was the Edge Split Cube in Cage. It has refractive index -2.7 (Fig. 2B) at $170 \mathrm{THz}$ and maximal figure of merit 2.3. It shows the Drude-like permittivity with an antiresonance in the negative index region (Fig. 3B) and negative permeability (Fig. 4B). Despite of the success it was obvious that such design can hardly be fabricated due to a big number of fine details and small slits at the edge of the Cube. Another problem was that ESCiC looses negative index properties for several layers. All that stimulated us to optimize the nested structure, and we came up with the Split Cube in Carcass.

One layer of SCiC has minimal refractive index -1.5 (Fig. 2C) and maximal figure-of-merit 2 at $187 \mathrm{THz}$. Effective permittivity (Fig. 3C) obeys Drude's law and reminds the permittivity of the bulk silver but reduced in magnitude by 10 times. The antiresonance phenomenon still presents in the spectra, but less pronounced in comparison with the ESCiC. Magnetic permeability (Fig. 4C) shows resonant behaviour and reaches -0.4 with its real part. 

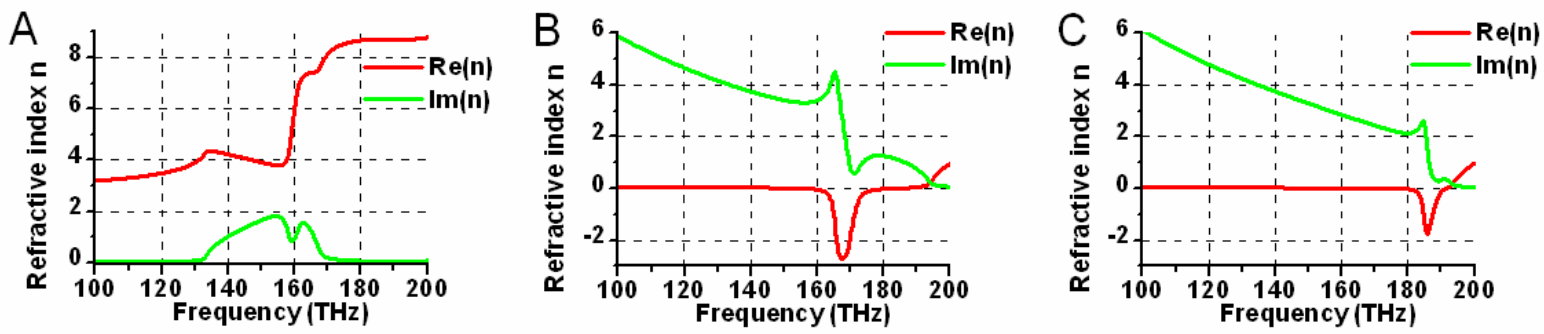

Figure 2. Effective refractive index of one layer of $\mathrm{CaP}(\mathrm{A}), \mathrm{ESCiC}(\mathrm{B})$ and $\mathrm{SCiC}(\mathrm{C})$
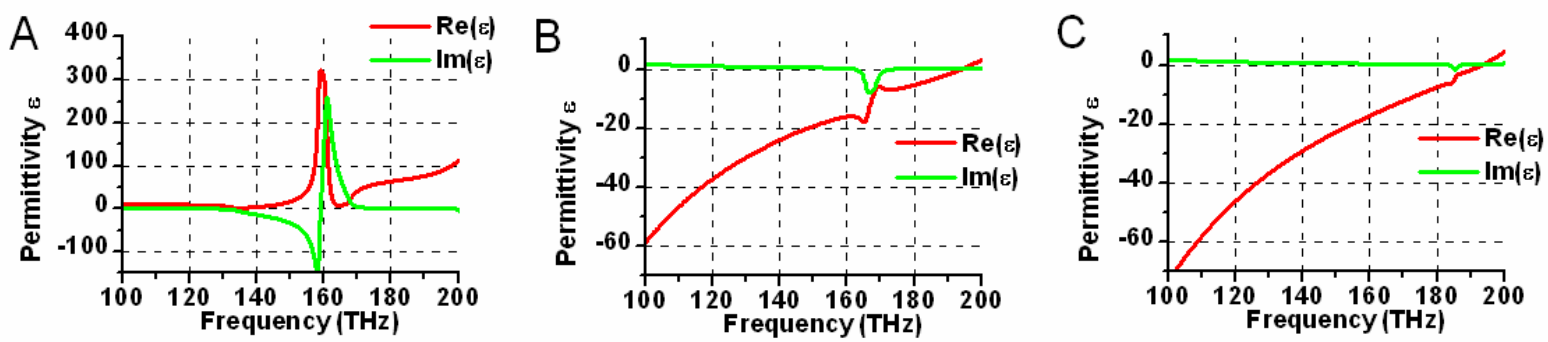

Figure 3. Effective permittivity of one layer of CaP (A), ESCiC (B) and SCiC (C)
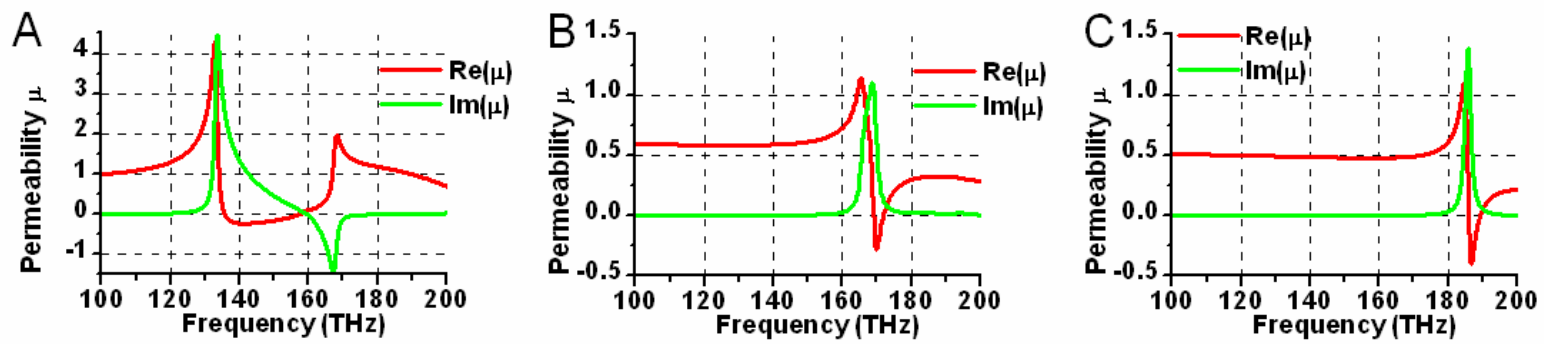

Figure 4. Effective permeability of one layer of CaP (A), ESCiC (B) and SCiC (C)

\subsection{Split Cube in Carcass - bulk isotropic NIM}

Split Cube in Carcass design shows fast convergence of the effective properties with the number of layers (Fig. 5). The values of its effective parameters do not change after 3 layers, so MTM exhibit behaviour of a bulk material.

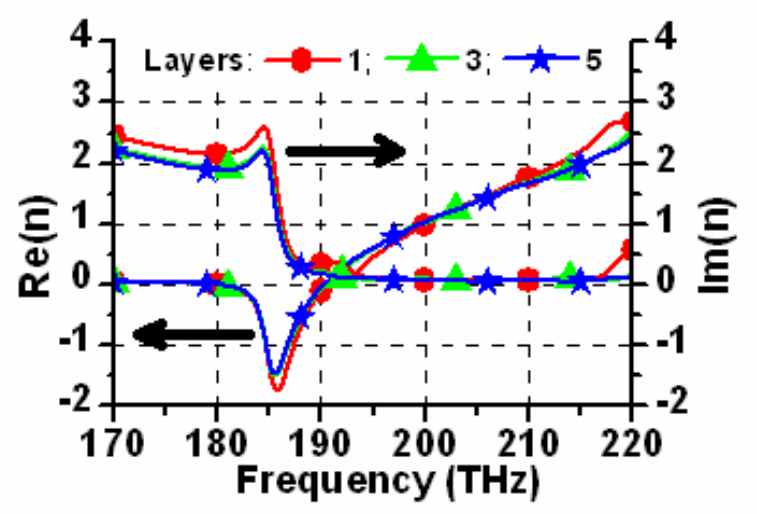

Figure 5. Effective refractive index of 1, 3 and 5 layers of SCiC

To confirm an isotropic response of our design in the telecom range we performed a bands dispersion analysis. The dielectric constant was chosen $\varepsilon=-140+0 i$, in correspondence with the bulk silver parameters in the region of interest. Losses were neglected for the purpose of simplifications.

Band structure analysis shows that there are several bands with almost linear dispersion. Isofrequency surfaces calculated for two degenerate modes at the homogeneity limit $k=\pi / 2 a$ were very close to the spherical shape (Fig. 6A). Further we plot bands dispersion (Fig. 6B). For the lower band eigenfrequencies for all three 
principle directions of the simple cubic lattice: $\Gamma-\mathrm{X}, \Gamma-\mathrm{M}$ and $\Gamma-\mathrm{R}$, almost coincide. So, following the arguments presented in [8] we regard this coincidence in eigenfrequencies enough to claim NIM's isotropy. This means that Split Cube in Carcass is indeed isotropic negative index material.
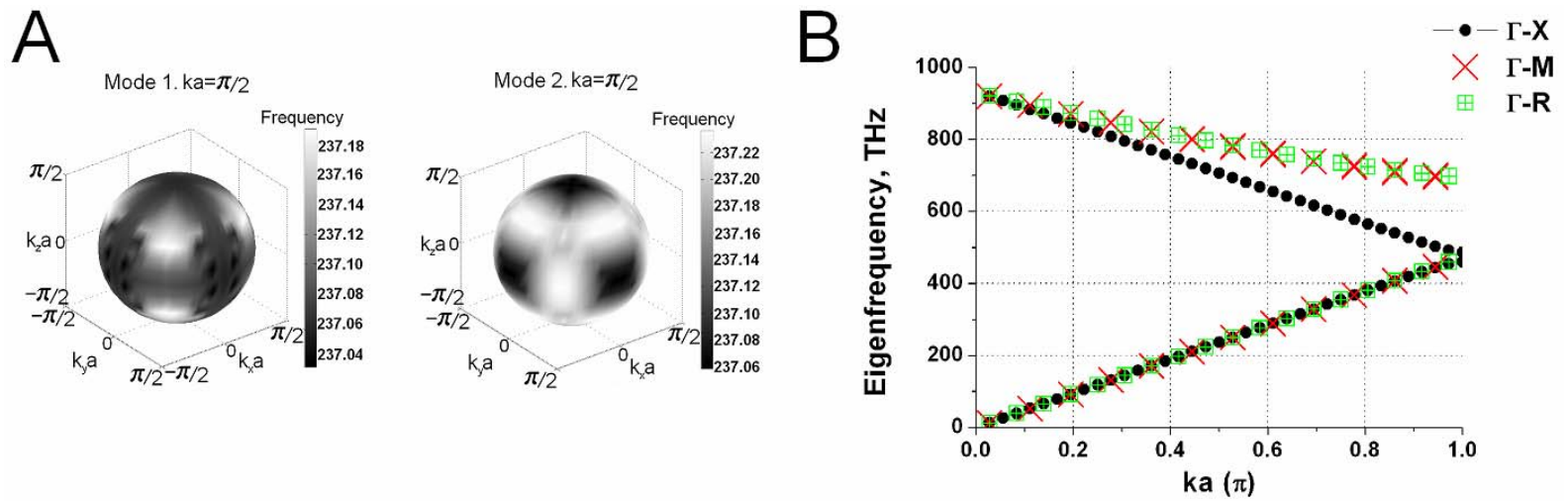

Figure 6. Frequency surface for $k a=\pi / 2$ (A) and dispersion diagram for $\Gamma-X, \Gamma-M, \Gamma$-R directions (B) for Split Cube in Cage.

\section{CONCLUSIONS}

We analysed three different designs of unit cell aiming for the isotropic bulk metamaterial in the infrared range. The evolution of our approach within the cubic symmetry constrain of the unit cell helped to achieve negative index response at $187 \mathrm{THz}$ in combination with a reasonable FOM. Nested structures concept was a key to reach the isotropic behaviour of the structure with proposed Split Cube in Carcass design. The fast convergence of retrieved effective parameters (after 3 layers changes are negligible) points to the bulk character of the metamaterial on the base of the SCiC unit cell. The special check for the exhibiting isotropy was done with the help of band dispersion analysis applying some fixed values of dielectric constant of the metal without losses. Its high transmission together with an isotropy which comes from a cubic symmetry of the unit cell makes SCiC a promising candidate for infrared and THz applications.

\section{ACKNOWLEDGEMENTS}

The authors gratefully acknowledge partial supports from the Danish Research Council for Technology and Production Sciences via the NIMBus project and COST Action MP0702.

\section{REFERENCES}

[1] V.G. Veselago: The electrodynamics of substances with simultaneously negative values of $\varepsilon$ and $\mu$, Sov. Phys. Usp., vol. 10. pp. 509-514, 1968.

[2] J.B. Pendry: Negative refraction makes a perfect lens, Phys. Rev. Lett., vol. 85. pp. 3966-3969, 2000.

[3] A. Degiron et al.: Negative index and indefinite media waveguide couplers, Appl. Phys. A, vol. 87. pp. 321-328, 2007.

[4] D.R. Smith et al.: Composite medium with simultaneously negative permeability and permittivity, Phys. Rev. Lett., vol. 84. pp. 4184-4187, 2000.

[5] J. Valentine et al.: Three-dimensional optical metamaterial with a negative refractive index, Nature, vol. 455. pp. 376-379, 2008.

[6] D.R. Smith et el.: Determination of effective permittivity and permeability of metamaterials from reflection and transmission coefficients, Phys. Rev. B, vol. 65. pp. 195104, 2002.

[7] A. Demetriadou, J.B. Pendry: Taming spatial dispersion in wire metamaterial, Journ. of Physics: Cond. Matter, vol. 20. pp. 295222, 2008.

[8] A.K. Iyer, G.V. Eleftheriades: A three-dimensional isotropic transmission-line metamaterial topology for free-space excitation, Appl. Phys. Lett., vol. 92. pp. 261106, 2008. 